\title{
Educational Knowledge Management Systems Model Based on Web 3.0 Technology
}

\author{
Prof. Mohamed M. El Hadi \\ Sadat Academy for \\ Management Sciences
}

\author{
Ahmed M. Amer \\ Ph.D. Candidate \\ at SAMS
}

\section{ABSTRACT}

Educational knowledge management systems based on Web 3.0 technologies are a modern approach to educational institutions, a new way of teaching and learning as well as for creation of own knowledge in virtually or real teaching and learning environment. Web 3.0 is described as integrate semantic web, big data, cloud computing, 3D visualization, mobility; intelligent agents based on semantic machines, and distributed artificial intelligence into the ubiquitous networks, leading to convenient access quickly to information for successful educational knowledge management. Web 3.0 tools such as semantic Web, semantic search, semantic social information spaces, Semantic forums, Semantic search, Semantic Wikis are able to effectivelycreate, capture, refine, store, manage, connect and disseminate Knowledge across the educational institutions to make teaching and learning more effective. This creates a method of learning a lot different from conventional methods commonly used in teaching and learning. This paper aims to present knowledge management systems model in educational institutions by using Web 3.0 technologies

Keywords: knowledge Management Systems, Educational Knowledge Management Systems, Web 3.0 Technologies, Semantic Web Tools, Knowledge Extraction, Educational Institutions.

\section{Introduction:}

The most important mission of a university as an educational organization is to provide its students with good learning environment. The concept of knowledge management is important not only in industries but also in such an educational organization so that it can manage the data concerning its students' learning ability, willingness to study, and other aspects that relate to learning and studying.( Minami, 2013).Knowledge Managementis a multidisciplinary approach of achieving organizational objectives by making the best use of knowledge. It focuses on processes such as acquiring, creating and sharingknowledge and the cultural and technical foundations that support them (Kundus,2013). Any kind of IT system that stores and retrieves knowledge, improves collaboration, locatesknowledge sources, mines repositories for hidden knowledge, captures and uses knowledgeor in some other way enhances the KM process (Froset, 2014).

The 2011 edition of the Horizon Report (Johnson et al., 2011) predicts thatthe Semantic Web will become more prevalent and prominent in educational settings in the next four to five years (Czerkawski, 2012). The use of Semantic Web technologies instead of knowledge structures or fuzzy sets approaches offers a number of advantages (Abbas, Ahmadand Kalid, 2014). Nowadays, Web 3.0seems to be the predominant technology. There is agreat interest of research regarding Web basedlearning (Albu, 2014).

\section{Knowledge Management:}

The knowledge management literature covers a 
wide range of factors affecting the creation, sharing, transfer, and acquisition of knowledge. These factors center around three basic knowledge entities - knowledge workers, knowledge processes, andknowledge tools. The breadth of literature examines how these entities interrelate and areaffected by variations in the operational environment. Existing research in knowledgetools focuses on specific attributes - technology, usability, or administration (Varghese, 2014).

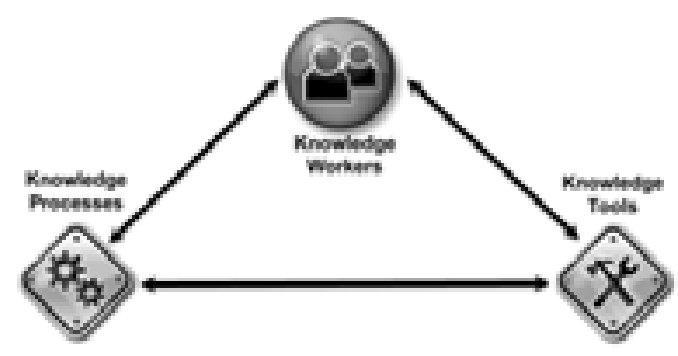

Figure 1. Elements of knowledge management

As indicated in Figure 1, each of knowledge workers, knowledge processing and knowledge tools elements are interrelated. Knowledgeworkers and knowledge processes address questions of who and what, while knowledgetools, also referred to a knowledge management systems (KMS), address how. Knowledge workers are the individual performing the knowledge management activity. Knowledge processes are the policies and procedures that define how KM can beperformed. Knowledge tools provide the technological foundation to workers andprocesses that aid in KM performance (Wan, Guo, Hu and Zeng, 2011).

\section{Knowledge Management Processes:}

Knowledge management processes are knowledge capture andcreation, knowledge organization and retention, knowledge dissemination, and knowledge utilization (Anand and Singh, 2011). Knowledge management processes are:knowledge creation, knowledge up-gradation, knowledgedisseminationandknowledgeRetention (Mishra and Baskar, 2011). Knowledge management implementation should addresses the five basic processes of knowledge management: knowledge acquisition, knowledge storage, knowledge dissemination and knowledge application (Cob, Abdullah, Risidi and Nor, 2015).

\section{Knowledge Management Systems:}

Some basic definitions of knowledge management systems are:

Knowledge management ( $\mathrm{KM}$ ) system is a collective term that is used to describe the creation of knowledge repositories, respective interface components, improvement of knowledge access and sharing as well as communication through collaboration, enhancing the knowledge environment and managing knowledge as an asset for an organization (Subramanian, Geetha, Mehata, and Hussain, 2012).

A system (generally information technology based) for managing knowledge in organizations for supporting thecreation, capturing, storage and dissemination of information. It can comprise a part (neither necessary nor sufficient) of a KM initiative(Yukikaze, 2012).

Any kind of information technology system that stores and retrieves knowledge, improves collaboration, locatesknowledge sources, mines repositories for hidden knowledge, captures and uses knowledge orin some other way enhances the KM process (Frost, 2014).

\section{Educational Knowledge Management:}

Educational knowledge management among the relevant organizations is quite important. This means "knowledge in universities circulated-systematic process" of finding, selecting, organizing, distilling and presenting information in a way that improves a learner's competency and/or ability to fulfill his or her necessary learning objectives (Okamoto, Nagata, and Anma, 2009).

Educational Knowledge Management is a process, making the continual creation and transmission of knowledge and wisdom in the whole education, in which it digitizes, cures, stores and disseminates the knowledge using technology (Wan, Guo, Hu, and Zeng, 2011). The process of educational knowledge management will be mainly automated, and this will increase the amount and accuracy of the data that can be processed into knowledge. In the meanwhile, (Thorn, 2001) articulates four primary goals of Educational Knowledge Management Systemsas follows:

(1) to create knowledge repositories, 
(2) to improve access to knowledge,

(3) to enhance the educational environment through knowledge sharing, and

(4) to manage knowledge as an asset to make sure that it contributes to bottom line success (Spector, Merrill, Elen, Bishop, 2014). .

\section{Web 3.0 Definition:}

Web 3.0, a phrase coined by John Markoff of the New York Times in 2006, and refers it to as a supposed third generation of Internet-based services that collectively comprise what might be called the intelligent Web'-such as those using semantic web, microformats, natural language search, data mining, machine learning, recommendation agents, and artificial intelligence technologies. Such this definition emphasizes machine-facilitated understanding of information in order to provide a more productive and intuitive user experience.

Nova Spivack defines also Web 3.0 as the third decade of the Web (2010-2020) during which he suggests several major complementary technology trends will reach new levels of maturity simultaneously including:

- Transformation of the Web from a network of separately silo applications and content repositories to a more seamless and interoperable whole.

- Ubiquitous connectivity, broadband adoption, mobile Internet access and mobile devices.

- Network computing, software-as-a-service business models, Web services interoperability, distributed computing, grid computing and cloud computing.

- Open technologies, open APIs and protocols, open data formats, open-source software platforms and open data (e.g. Creative Commons, Open Data License).

- Open identity, OpenID, open reputation, roaming portable identity and personal data.

- The intelligent web, Semantic Web technologies such as RDF, OWL, SWRL, SPARQL, GRDDL, semantic application platforms, and statement-based data stores.

- Distributed databases, the "World Wide Database" (enabled by Semantic Web technologies.

- Intelligent applications, natural language processing, machine learning, machine reasoning, and autonomous agents. (Spivack, 2015)

\subsection{Technology used in Web 3.0:}

a. Semantic Web:The phrases Semantic Web and Web 3.0 are often used interchangeably. Semantic web serves as a place, where machines will be able to read web pages much like humans, and search engines along with software agents will troll the Internet to find and show what you are exactly looking for. It is all about representing meanings, connecting knowledge.

b. Intelligent Web: Web 3.0 is based on "intelligent" web applications using:Natural language processing, Machine-based learning and reasoning, Intelligent applications, and Openness - Open user ID, roaming portable identity and Personal Data.

c. World Wide Database: Nova Spivack defines Web 3.0 as "a set of standards that turns the Web into one big database." Web 3.0 uses structured data records which are published to the Web in reusable and remote-queriable formats.

d. 3D Web: Web 3.0 will use a three dimensional model and transform it into a series of 3D spaces. Web 3.0 promises: bandwidth-heavy content such as 3D, audio and video, heavily interlinked services, highly localized and personalized services, or Web 3.0 3D interactive technology, and the motivation is real time interaction and learning as a result of visualization and collaboration.

e. Social Web: Concepts such as social networking, social bookmarking and in-group searching will produce a much more customized and targeted Web surfing experience. A single login will allow you to set your status update on Facebook, Twitter and MySpace together.

f. Interoperability: Web 3.0 allows users to roam freely from database to database, program to program, and device to device.

g. Mobility: Web 3.0 is all about ubiquitous connectivity, broadband adoption, mobile Internet access, mo- 
bile devices, compatibility with any device, computer, mobile phone or even TV and fast and customizable applications.

\subsection{Semantic Web Technologies:}

Standardization for Semantic Web in the context of Web 3.0 is under the care of W3C. The term "Semantic Web" is often used more specifically to refer to the formats and technologies that enable it. The collection, structuring and recovery of linked data are enabled by technologies that provide a formal description of concepts, terms, and relationships within a given knowledge domain. These technologies are specified as W3C standards and include: (Semantic Web, 2015)

- Resource Description Framework (RDF), a general method for describing information

- RDF Schema (RDFS)

- Simple Knowledge Organization System (SKOS)

- SPARQL, an RDF query language

- Notation3 (N3), designed with human-readability in mind

- N-Triples, a format for storing and transmitting data

- Turtle (Terse RDF Triple Language)

- Web Ontology Language (OWL), a family of knowledge representation languages

- Rule Interchange Format (RIF), a framework of web rule language dialects supporting rule interchange on the Web

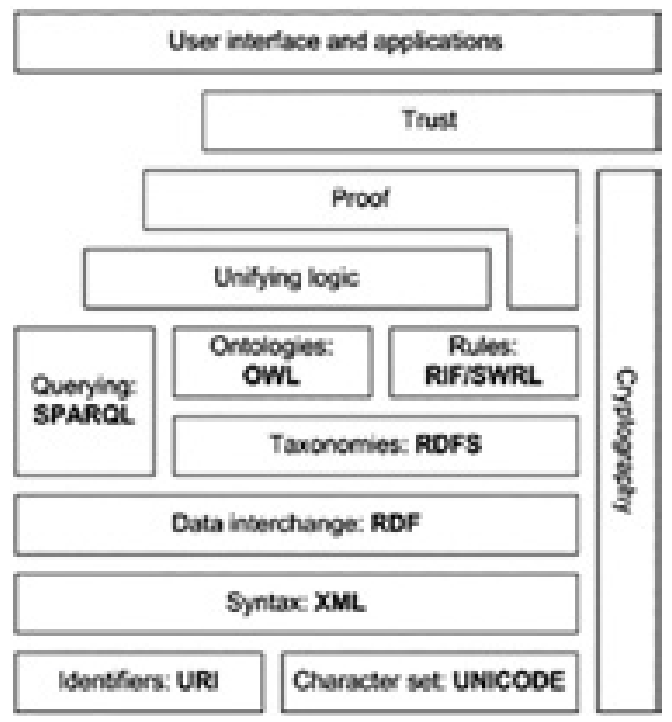

Figure 2.The Semantic Web Stack
The Semantic Web Stack illustrates the architecture of the Semantic Web. The functions and relationships of the components can be summarized as follows:

- XML provides an elemental syntax for content structure within documents, yet associates no semantics with the meaning of the content contained within. $\mathrm{XML}$ is not at present a necessary component of Semantic Web technologies in most cases, as alternative syntaxes exists, such as Turtle. Turtle is a de facto standard, but has not been through a formal standardization process.

- XML Schema is a language for providing and restricting the structure and content of elements contained within XML documents.

- RDF is a simple language for expressing data models, which refer to objects ("web resources") and their relationships. An RDF-based model can be represented in a variety of syntaxes, e.g., RDF/XML, N3, Turtle, and RDFa. RDF is a fundamental standard of the Semantic Web.

- RDF Schema extends RDF and is a vocabulary for describing properties and classes of RDF-based resources, with semantics for generalized-hierarchies of such properties and classes.

- OWL adds more vocabulary for describing properties and classes: among others, relations between classes (e.g. disjointness), cardinality (e.g. "exactly one"), equality, richer typing of properties, characteristics of properties (e.g. symmetry), and enumerated classes.

- SPARQL is a protocol and query language for semantic web data sources.

- RIF is the W3C Rule Interchange Format. It's an XML language for expressing Web rules which computers can execute. RIF provides multiple versions, called dialects. It includes a RIF Basic Logic Dialect (RIF-BLD) and RIF Production Rules Dialect (RIF PRD).

\subsection{Semantic Tools}

Semantic tools are designed to support semantic functionalities which can deploy in order to support semantic features; these semantic solutions can be cate- 
gorized based on their purpose and function it supports as under: (Pandey and Panda, 2014)

a. RDF conversion/Visualization Tools: Used for documenting and explaining RDF mapping, e.g. PoolParty Extractor.

b. Treasures Knowledge Organization Systems Tools: Used for representing and sharing knowledge organization systems over the web, e.g. SKOS.

c. Metadata Schema/Standards: Helps in transforming a flat metadata schema to semantic web ontology, e.g. MADS.

d. Supportive Tools - Plug-in: Helps to add schema which adds a specific feature to an existing application, e.g. Zotero Firefox Plugin.

e. Fully Flashed Project/Separate portal: These projects/applications incorporate features of semantic search and browse as well as follows semantic architectural models for storage of information, e.g. Semantic Medline.

f. Open Source - Java Software/Program/ Searching Tools: these tools serve different purposes of semantic applications from providing standard terminology to searching, e.g. GoNTogle and semantic personal digital library and RelFinder.

g. Interoperability/ Harvesting Tools: Interoperability toolenables application to communicate with other applications at cross-linguistic interoperability and metadata interoperability levels.e.g. JSTOR/Harvard Object Validation Environment (JHOVE).

h. Knowledge Extraction Tools: Helps in creation of knowledge from structured and unstructured sources, e.g. PoolParty Extractor.

i. Ontology engineering Tools: Any tool used for creating ontologies or semantic document, e.g. semanticMediaWiki.

j. Semantic Measures Tools: Used for computation and analysis of semantic measures, e.g. semantic similarity, semantic relatedness, semantic distance, etc.

Summarizes some of the Knowledge Extraction tools e.g. in a comprehensive way as follows:

a. AIDA is a framework and online tool for named en- tity recognitionand disambiguation. It consults YAGO2 ontology with the aim tomap mentions of source document text with disambiguated entities ofYAGO2 knowledge-base.

b. DBpedia Spotlighttool is developed for automatically annotatingmentions of DBpedia resources in the text.

c. AlchemyAPlaims to extract named entities, their relationships, topics and sense tagging by analyzing web or text-based content usingparsing and machine learning. It does not provide a direct RDF en-coding.

d. CiceroLitealso called Extractiv, recognizes named entities for English, Arabic, Chinese and some European-language texts. It also performs relation extraction, semantic role labeling and sense tagging.

e. NERDis merger of knowledge extraction tools (AlchemyAPI, DB-pedia Spotlight, Extractiv, Lupedia, OpenCalais, Saplo, SemiTags, Wikimeta, Yahoo! Content Analysis, and Zemanta) focusing sense tagging and named entity recognition and resolution.

f. Wikimetaperforms sense tagging and recognizes named entities anddisambiguate them. Text data is linked to concepts of the LinkedOpen Data network through different sources like Geonames, DB-pedia, Wikipedia or CIA World Factbook or the web on unavailabilityof any resource.

g. FOXfocuses sense tagging, named entity recognition and resolution, term and relation extraction.

h. FREDautomatically produces RDF/OWL ontologies and linked datafrom text based on deep semantic parsing, discourse representationtheory, linguistic frames and ontology design patterns. SemiosearchWikifierproduces NER results.

i. Zemantahas interaction capabilities where it matches text with pub-licly available content and produces it in the creation tool as it is beingwritten. It also performs content linking and recognizes named entities and disambiguate them.

j. PoolParty Knowledge Discovererperforms text mining and recognizes named entities based on knowledge models, thesauri and linkeddata. It depends upon 
a reference knowledge-base derived from somecontrolled vocabularies such as thesaurus. Images, tags, content andcategories are recommended automatically when controlled vocabularies are used as a base knowledge model.

k. Open Calaisis a knowledge extraction tool used for named entityrecognition with sense tags, facts and events..

I. ReVerbautomatically identifies and extracts binary relationships fromEnglish sentences. It runs on a model trained out of the big dataset ofOpen Information Extraction web triples. It takes raw text as input andproduces triples (argument1, relation phrase, argument2) as output. Itdoes not work on bulk text.

m. Apache Stanbolsemi-automatically enhances unstructured text withsemantic annotations to be able to link documents with related entities and topics. Current enhancers include RDF encoding of resultsfrom multilingual named entity recognition and resolution, sense tagging with reference to DBpedia and GeoNames and related images etc.

n. SemiosearchWikierintegrates various components i.e., a namedentity recognizer (currently Alchemy), a semiotically informed indexof Wikipedia pages, as well as matching and heuristic strategies forresolving arbitrary named entities or terms on DBpedia entities.

5.5 Tools and Services of Web 3.0 for teaching and learning

Some of the Web 3.0 tools and services which are useful for the education and research are learner centered Technologies, knowledge representation, extended smart mobile technology, distrusted computing, collaborative intelligent filtering, ubiquitous learning, peer-learning group, collaborative learning, 3d Visualization and Instruction and adaption.We describe briefly some of the Web 3.0 tools and services: (Rajiv and Lal, 2011)

a. Learning with 3D-Wikis / Virtual 3D Encyclopedia: 3D Wikis would be able to providerich \& effective environment involving all media andanimation, for learners, so that they can have better impact onlearning \& knowledge. b. Learning with 3D Virtual worlds \& Avatars: As mentioned earlier, a 3D virtual world is a mix of 3D gamingtechnology, augmented reality, simulated environment poweredwith Internet technology where users interact through movableavatars.

c. Intelligent Search Engines: Students will benefit fromknowledge construction powered by the Semantic Web. Ontologies will link the learner's needs and characteristics so that personalized agentscan search for learning material based on the learners' needs.

d. Online 3-D Virtual Labs / Educational labs / Simulations or 3D Web: 3D rich graphical user interfaces will act as a powerfulplatform for the users to participate and perform collaborativeactivities, sharing results and exchanging media informationamong participants in a more natural way.

\section{EducationalKnowledge Management System} Architecture:

The architecture of EKMS model depicted in figure 3 consists of six layers as below:

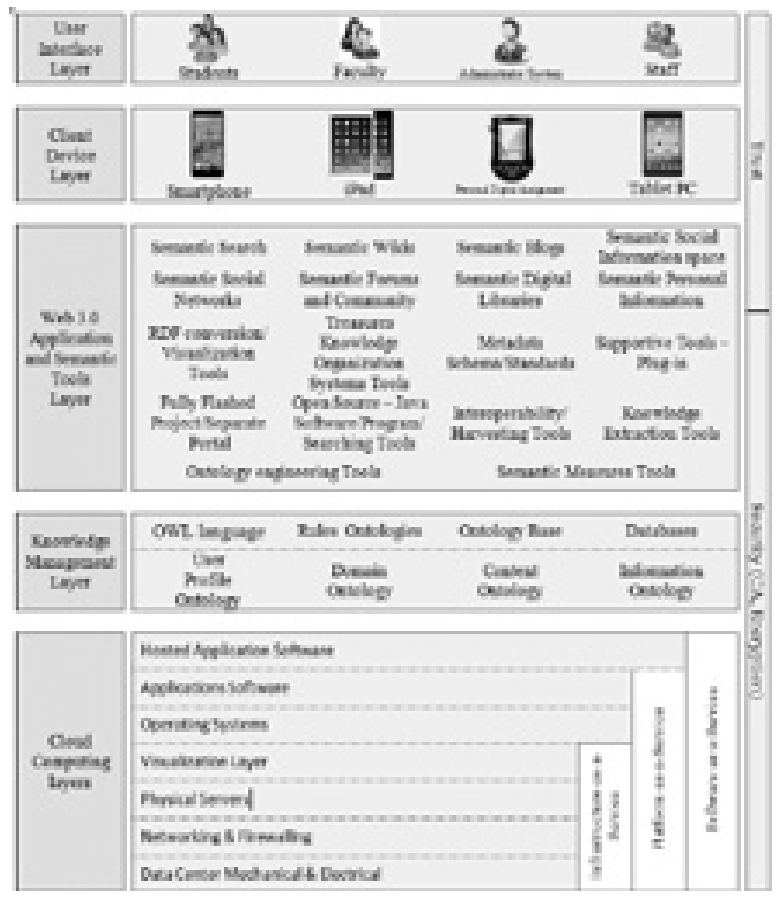

Figure 3. The architecture of educational knowledge management systems

Model- based on Web 3.0 technologies

Cloud Computing Layers. The cloud computing layers 
as below:

- SaaS: The upper layer is software as a service that letto user to run different applications from the cloud.

- PaaS: Platform as a Service provides the operativesystem for application that implement on the cloud.

- laas: This layer offers the storage and computingresource as a service. The goal ofthis layer is similar toPaas while this layer refers to hardware.

Knowledge Management Layer. The knowledge layer is located between the cloud computing layer and Web 3.0 application and tools layer OWL language is prevailingtechnologies in the knowledge management layer.

Web 3.0 Applications and Semantic Tools Layer. The layer combinesWeb 3.0 and web2.0 with semantic tools layer.

Trust and Security Layer. The layer becomes a primary boundary of access control for security requirements as well.

Client Device Layer. The client device layer such as tablets, smartphones and iPad. Users access using client devices.

User Interface Layer. User Interface layer is also called presentation layer. The user interface layer is the top of layer in the architecture; this layer defines how the user directly interacts with an EKMS.

\section{Conclusion:}

The biggest challenges in educational institutions are collecting information and manage knowledge. Educational institutions need to adopt modern technology in the implementation of educational knowledge management systems. The web 3.0 is the new technologies. Web 3.0 technologies give the educational knowledge management systems powerful tools to manage knowledge. The educational knowledge management systems model includes six layers: cloud layer, knowledge management layer, semantic tools layer, semantic Learning Web application layer,access layer, and user interface layer, each layer includes various services and tools. Web 3.0 technologies are still in the developing stage.Web 3.0 will change the way of students and teachers interact with mobile devices, and networks e.g. internet, intranet, extranet. Students and teachers can access the educational knowledge management system via a variety of devices, such as mobile phones, iPad, Personal Digital Assistants (PDAs) and Tablet PC. etc. . The model of EKMS based on Web 3.0 technologies consists of four key elements: knowledge management, Web 3.0 technologies, Staff, and educational institutions.

\section{References}

Abbas,M. A., Ahmad,W. F. W. \&Kalid K. S. (2014). Semantic Web Technologies for Pre-School Cognitive Skills Tutoring System, Journal of Information Science and Engineering 30,pp. 835-851.

Albu, Razvan-Daniel. (2014). A Study about Web 3.0 based E-Learning Tools in Electrical and Electronics Engineering, Journal of Electrical and Electronics Engineering, Vol. 7, Issue 1, p.9.

Anand, A., \& Singh, M. D. (2011). Understanding knowledge management: A literature review. International Journal of Engineering, Science and Technology, Vol. 3, No. 2 , pp. 926-939.

Cob Z., Abdullah R., Risidi H., and Nor M. (Eds). (2015). Preliminary study on semantic knowledge management model for collaborative learning, ARPN Journal of Engineering and Applied Sciences, Vol. 10, No. 2,pp. 442-450.

Czerkawski, B. (2012). Benefits and Potentials of Semantic Web in Teacher Education Programs. In P. Resta (Ed.), Proceedings of Society for Information Technology \& Teacher Education International Conference 2012, pp. 3581-3583.

Frost, Alla.(2014). KnowledgeManagement Tools: What is KnowledgeManagement? http://www.knowledgemanagement-tools.net/knowledge-management. html,Viewed 25/4/2015.

Johnson, L., Smith, R., Willis, H., Levine, A., \& Haywood, K., (2011). The 2011 Horizon Report. Austin, Texas: The New Media Consortium.

Kundus, J.K. (2013). Knowledge Management: Theory and Application, Tiwala publishing: New Delhi.

Minami, T. ,(2013). Profiling of Patrons' Interest Areas from Library's Circulation Records - An Approach to Knowledge Management for University Students, The Fifth International Conference on Information, Process, and Knowledge Management, IARIA, pp. 45-50. 